\title{
Effect of the Primary User Traffic on Cognitive Relaying with Adaptive Transmission
}

\author{
Anlei Rao*, Hao Ma*, Mohamed-Slim Alouini* and Yunfei Chen ${ }^{\dagger}$ \\ ${ }^{*}$ Division of Physical Sciences and Engineering, KAUST \\ Email: \{anlei.rao, hao.ma, slim.alouini\}@kaust.edu.sa \\ ${ }^{\dagger}$ School of Engineering, University of Warwick \\ Email: yunfei.chen@warwick.ac.uk
}

\begin{abstract}
In a cognitive-relay system, the secondary user is permitted to transmit data via a relay when the spectrum bands are detected to be free. The miss detection of spectrum sensing and the primary user traffic will affect the data transmission performance of the secondary user. In this paper, we investigate the impact of the status change of the primary user on the bit error rate (BER) of the adaptive transmission of the secondary user in a cognitive-relay system. Numerical results show that the primary user traffic can significantly degrade the BER of the secondary user transmission.
\end{abstract}

\section{INTRODUCTION}

Due to the dramatic growth of wireless services over the last decades, cognitive radio has been proposed as a revolutionary technology to solve the conflict between spectrum scarcity and spectrum under-utilization [1]. When the licensed bands in the spectrum are detected to be unoccupied, the secondary users could utilize these free bands to perform data transmission. In a multiuser environment, each secondary user has a chance to be chosen by the base station to transmit data over the free spectrum bands. The selected secondary user, however, may unfortunately suffer from severe fading or shadowing. In this case, any other secondary user with better channel conditions may act as a relay for data transmission.

There are mainly two kinds of methods for relaying: amplify-and-forward (AF) and decode-and-forward (DF) methods. In the AF method, the relay receives the signals from the transmitter, and then simply amplifies the signals and retransmits them. In the DF method, the relay first decodes the received signals and then re-encodes and re-transmits them [2]. In [3, 4], the authors used the AF relaying method for cooperative spectrum sensing. In our case, the relay acted by the secondary user for data transmission also adopts the AF method.

Adaptive modulation [5] is an effective method widely used to increase the link spectral efficiency. When the channel side information can be estimated and this estimation sent back to the transmitter, the transmitting rate and power can be adapted relative to the channel characteristics to achieve better performance. With adaptive modulation, the secondary user could perform data transmission with more flexibility and higher efficiency. One problem under this framework is that the primary user may randomly come or leave during the data transmission period of the secondary users. This will certainly affect the performance of adaptive modulation due to the mismatch of the channel conditions. In this paper, we study the effects of the primary user traffic on the bit error rate (BER) of adaptive transmission of the secondary user in a cognitive-relay system.

The rest of the paper is organized as follows. In Section II, we introduce the basic assumptions and models for cognitive adaptive transmission with a relay. Then in Section III, the conditions with initially present and absent primary user are analyzed, and we show the results exerted by the impact of the primary user traffic on average BER over each link. Next, Section IV presents different scenarios of the primary user status change and gives the average BER over all possible scenarios. Finally, Section V shows some numerical results and the conclusions are drawn in the last section.

\section{SySTEM MODEL}

In a cognitive-radio framework, data transmission can be performed on a frame-by-frame basis, where each frame includes the overhead part for spectrum sensing and the data part for transmission. For spectrum sensing, we assume that the false-alarm probability and miss-detection probability are given by $P_{f a}=\operatorname{Pr}\left\{H_{1} \mid H_{0}\right\}$ and $P_{m d}=\operatorname{Pr}\left\{H_{0} \mid H_{1}\right\}$, where $H_{0}$ and $H_{1}$ denotes the absence and presence of the primary user, respectively. When the primary user is detected to be absent, the secondary users can use the free spectrum bands to perform the adaptive transmission.

Under severe fading and shadowing, the selected secondary user can transmit with a relay. We denote $\gamma_{1}$ as the signal-tonoise ratio (SNR) for the channel between the transmitter and relay, or the source-relay $(S-R)$ link, and $\gamma_{2}$ as the SNR for the channel between relay and receiver, or the relay-destination $(R-D)$ link. For Nakagami fading channels, the probability density function (PDF) of $\gamma_{1}$ and $\gamma_{2}$ are given by [6]

$f_{\gamma_{i}}(\gamma)=\left(\frac{m_{i}}{\bar{\gamma}_{i}}\right)^{m_{i}} \frac{\gamma^{m_{i}-1}}{\Gamma\left(m_{i}\right)} \exp \left(-\frac{m_{i}}{\bar{\gamma}_{i}} \gamma\right), \quad \gamma>0, i=1,2$,

where $\bar{\gamma}_{i}$ and $m_{i}$ are the mean and Nakagami parameter for $\gamma_{i}$. By properly choosing the relay gain, the end-to-end SNR 


$$
M_{Z}(s ; a, b)= \begin{cases}\mathrm{e}^{s / b}\left(\frac{\beta_{2} b}{\beta_{1} a}\right)^{\alpha_{1}} \Phi_{1}\left(\alpha_{1}, \alpha_{1}+\alpha_{2}, \alpha_{1}+\alpha_{2} ; 1-\frac{\beta_{2} b}{\beta_{1} a}, \frac{b-a}{a b} s\right), & 0<\frac{\beta_{2} b}{\beta_{1} a}<2, \\ \mathrm{e}^{s / a}\left(\frac{\beta_{1} a}{\beta_{2} b}\right)^{\alpha_{2}} \Phi_{1}\left(\alpha_{2}, \alpha_{1}+\alpha_{2}, \alpha_{1}+\alpha_{2} ; 1-\frac{\beta_{1} a}{\beta_{2} b}, \frac{a-b}{a b} s\right), & 0<\frac{\beta_{1} a}{\beta_{2} b}<2 .\end{cases}
$$

for the source-destination $(S-D)$ link is given by $[2,7]$

$$
\gamma=\frac{\gamma_{1} \gamma_{2}}{1+\gamma_{1}+\gamma_{2}}
$$

which can be well approximated by

$$
\gamma \approx \frac{\gamma_{1} \gamma_{2}}{\gamma_{1}+\gamma_{2}}
$$

Assuming perfect estimation of channel conditions and reliable feedback link and ignoring the time delay, we adopt adaptive modulation for the data transmission of the secondary user. With constant power allocation and uncoded M-ary quadrature amplitude modulation (M-QAM), the BER can be well approximated by [5]

$$
\operatorname{BER}(\gamma, M) \simeq 0.2 \exp \left\{-\frac{3 \gamma}{2(M-1)}\right\},
$$

where $\gamma$ is the instantaneous SNR and $M$ is the constellation size. To maintain a target operation BER, $\mathrm{BER}_{0}, M$ is adjusted according to the SNR $\gamma$ by

$$
M=1+\frac{3 \gamma}{2 K_{0}},
$$

where $K_{0}=-\log \left(5 \mathrm{BER}_{0}\right)$.

We assume that the primary user traffic follows an i.i.d. onoff process, and "0" and "1" are used to represent the case when the licensed channel is free and occupied, respectively. For each case, the holding time is assumed to be exponential with mean $\lambda$ for " 0 " and $\mu$ for " 1 ". So the status transition probability matrix is thus given by [8]

$$
\begin{aligned}
\boldsymbol{P} & =\left(\begin{array}{ll}
p_{00} & p_{01} \\
p_{10} & p_{11}
\end{array}\right) \\
& =\frac{1}{\lambda+\mu}\left(\begin{array}{ll}
\mu+\lambda \mathrm{e}^{-(\lambda+\mu) t} & \lambda-\mu \mathrm{e}^{-(\lambda+\mu) t} \\
\mu-\lambda \mathrm{e}^{-(\lambda+\mu) t} & \lambda+\mu \mathrm{e}^{-(\lambda+\mu) t}
\end{array}\right)
\end{aligned}
$$

The initial probability is given as $p_{b}=\frac{\mu}{\lambda+\mu}$ for busy licensed channel and $p_{f}=\frac{\lambda}{\lambda+\mu}$ for free licensed channel.

The data transmission periods for $S-R$ link and $R-D$ link take a total time of $M T_{s}$ and $N T_{s}$, respectively, where $T_{s}$ denotes the time of one symbol. We assume that the secondary frame is of approximately the same length as the primary frame such that the status of primary user changes at most once in each link.

\section{Initial Status OF THE PRIMARY USER}

\section{A. Mathematical Foundations}

Suppose $r_{1}$ and $r_{2}$ are two independent Gamma-distributed random variables with the scale parameter $\beta_{i}$ and shape parameter $\alpha_{i}$ such that $r_{i} \sim \mathcal{G}\left(\alpha_{i}, \beta_{i}\right), i=1,2$. Denote the random variable $Z$ as

$$
Z=\frac{r_{1}+r_{2}}{a r_{1}+b r_{2}}
$$

where $a$ and $b$ are any real-positive values. It is not difficult to show that the PDF of $Z$ is given by

$$
f_{Z}(z)=\frac{|a-b|}{\beta_{1}^{\alpha_{1}} \beta_{2}^{\alpha_{2}}} \frac{\Gamma\left(\alpha_{1}+\alpha_{2}\right)}{\Gamma\left(\alpha_{1}\right) \Gamma\left(\alpha_{2}\right)} \frac{(1-b z)^{\alpha_{1}-1}(a z-1)^{\alpha_{2}-1}}{\left(\frac{(1-b z)}{\beta_{1}}+\frac{(a z-1)}{\beta_{2}}\right)^{\alpha_{1}+\alpha_{2}}}
$$

The moment generating function (MGF) of $Z$ is shown as (9) on the top of this page with the help of the following integral $[9,3.385]$

$$
\int_{0}^{1} t^{u-1}(1-t)^{v-1}(1-x t)^{-\epsilon} \mathrm{e}^{y t} d t=B(u, v) \Phi_{1}(u, \epsilon, u+v ; x, y),
$$

where $u, v, \epsilon, x, y$ are any real numbers satisfying $u>0, v>$ $0,1>|x|>0, B(u, v)=\frac{\Gamma(u) \Gamma(v)}{\Gamma(u+v)}$ is the Beta function, and $\Phi_{1}(\cdot, \cdot, \cdot ; \cdot, \cdot)$ is the Humbert series.

\section{B. Primary User Initially Absent}

Without the presence of the primary user at the beginning, the constellation size is chosen from (5) for the data transmission of the secondary user. If the primary user arrives in the $S-R$ link, then there will be primary user traffic in the $S-R$ link. The $S-R$ link SNR may change from $\gamma_{1}$ to $\gamma_{1}^{\prime}=\frac{\gamma_{1}}{1+\gamma_{p}}$, where $\gamma_{p}$ is the SNR of the primary user signal, and the $S-D$ link SNR is thus given by

$$
\gamma_{A}^{\prime}=\frac{\gamma_{1}^{\prime} \gamma_{2}}{\gamma_{1}^{\prime}+\gamma_{2}}=\frac{\gamma_{1} \gamma_{2}}{\gamma_{1}+\left(1+\gamma_{p}\right) \gamma_{2}},
$$

The instantaneous operating BER yields

$$
\operatorname{BER}\left(\gamma_{A}^{\prime}, M\right) \simeq \frac{1}{5} \exp \left\{-\frac{3 \gamma_{A}^{\prime}}{2(M-1)}\right\}=\frac{1}{5}\left(5 \mathrm{BER}_{0}\right)^{x_{A}}
$$

where the random variable $x_{A}$ is defined as

$$
x_{A}=\frac{\gamma_{A}^{\prime}}{\gamma}=\frac{\gamma_{1}+\gamma_{2}}{\gamma_{1}+\left(1+\gamma_{p}\right) \gamma_{2}} .
$$

The average BER with primary user traffic in the $S-R$ link is thus given by

$$
\overline{\mathrm{BER}}_{1}=\int \frac{1}{5}\left(5 \mathrm{BER}_{0}\right)^{x} f_{x_{A}}(x) d x
$$

where $f_{x_{A}}(x)$ is the PDF of the random variable $x_{A}$.

For Nakagami fading channels, the PDFs for $S-R$ link SNR $\gamma_{1}$ and $R-D$ link SNR $\gamma_{2}$ are given in (1). Taking $a=1$ and $b=1+\gamma_{p}$, we have the random variable $Z$ in (7) yield $x_{A}$ in (13). With the help of the MGF of $Z$ in (9), we can rewrite $\overline{\mathrm{BER}}_{1}$ as

$$
\overline{\mathrm{BER}}_{1}=\frac{1}{5} M_{Z}\left(-K_{0} ; 1,1+\gamma_{p}\right) .
$$

where the parameters $\alpha_{i}$ and $\beta_{i}$ in (9) are given by $\alpha_{i}=$ $m_{i}, \beta_{i}=\bar{\gamma}_{i} / m_{i}, i=1,2$. 
If there is primary user traffic in the $R-D$ link, however, the $R-D$ link SNR may change from $\gamma_{2}$ to $\gamma_{2}^{\prime}=\frac{\gamma_{2}}{1+\gamma_{p}}$, and the $S-D$ link SNR is then given by

$$
\gamma_{B}^{\prime}=\frac{\gamma_{1} \gamma_{2}^{\prime}}{\gamma_{1}+\gamma_{2}^{\prime}}=\frac{\gamma_{1} \gamma_{2}}{\left(1+\gamma_{p}\right) \gamma_{1}+\gamma_{2}},
$$

Similarly, the instantaneous operating BER in this case yields

$$
\operatorname{BER}\left(\gamma_{B}^{\prime}, M\right)=\frac{1}{5}\left(5 \mathrm{BER}_{0}\right)^{x_{B}}
$$

where the random variable $x_{B}$ is defined as

$$
x_{B}=\frac{\gamma_{B}^{\prime}}{\gamma}=\frac{\gamma_{1}+\gamma_{2}}{\left(1+\gamma_{p}\right) \gamma_{1}+\gamma_{2}} .
$$

The average BER with primary user traffic in the $R-D$ link is thus given by

$$
\overline{\mathrm{BER}}_{2}=\int \frac{1}{5}\left(5 \mathrm{BER}_{0}\right)^{x} f_{x_{B}}(x) d x,
$$

where $f_{x_{B}}(x)$ is the PDF of the random variable $x_{B}$. Taking $a=1+\gamma_{p}$ and $b=1$, we have the random variable $Z$ in (7) yield $x_{B}$ in (18). With the help of the MGF of $Z$ in (9), we can rewrite $\overline{\mathrm{BER}}_{2}$ as

$$
\overline{\mathrm{BER}}_{2}=\frac{1}{5} M_{Z}\left(-K_{0} ; 1+\gamma_{p}, 1\right) .
$$

\section{Primary User Initially Present}

If the primary user is present at the beginning of the time slot, the estimation of the SNR for $S-R$ link and $R-D$ link should be $\gamma_{1}^{\prime}=\frac{\gamma_{1}}{1+\gamma_{p}}$ and $\gamma_{2}^{\prime}=\frac{\gamma_{2}}{1+\gamma_{p}}$, respectively. The $S-D$ link SNR then yields

$$
\gamma^{\prime}=\frac{\gamma_{1}^{\prime} \gamma_{2}^{\prime}}{\gamma_{1}^{\prime}+\gamma_{2}^{\prime}}=\frac{\gamma}{1+\gamma_{p}} .
$$

In this case, the secondary user is allowed to transmit only when miss detection happens for spectrum sensing. Under the assumption of miss detection, the constellation size for data transmission is thus chosen to maintain the operating BER unchanged as

$$
M^{\prime}=1+\frac{3 \gamma}{2 K_{0}\left(1+\gamma_{p}\right)},
$$

When the primary user leaves in the $S-R$ link, the instantaneous SNR will change back to $\gamma_{1}$, and the $S-D$ link becomes $\gamma_{B}^{\prime}$. In this case, the instantaneous BER yields

$$
\operatorname{BER}\left(\gamma_{B}^{\prime}, M^{\prime}\right)=\frac{1}{5}\left(5 \mathrm{BER}_{0}\right)^{x_{B}\left(1+\gamma_{p}\right)}
$$

And the average BER without primary user traffic in the $S-R$ link is thus given by

$$
\overline{\mathrm{BER}}_{3}=\int \frac{1}{5}\left(5 \mathrm{BER}_{0}\right)^{\left(1+\gamma_{p}\right) x} f_{x_{B}}(x) d x
$$

When the primary user leaves in the $R-D$ link, the instantaneous SNR will change back to $\gamma_{2}$, and the $S-D$ link becomes $\gamma_{A}^{\prime}$. Similarly, the average BER without primary user traffic in the $R-D$ link yield

$$
\overline{\mathrm{BER}}_{4}=\int \frac{1}{5}\left(5 \mathrm{BER}_{0}\right)^{\left(1+\gamma_{p}\right) x} f_{x_{A}}(x) d x
$$

Similarly with the help of (9), we can rewrite $\overline{\mathrm{BER}}_{3}$ and $\overline{\mathrm{BER}}_{4}$ as

$$
\begin{aligned}
\overline{\mathrm{BER}}_{3} & =\frac{1}{5} M_{Z}\left(-\left(1+\gamma_{p}\right) K_{0} ; 1+\gamma_{p}, 1\right), \\
\overline{\mathrm{BER}}_{4} & =\frac{1}{5} M_{Z}\left(-\left(1+\gamma_{p}\right) K_{0} ; 1,1+\gamma_{p}\right) .
\end{aligned}
$$

\section{Performance Analysis}

As we stated above, the secondary user could transmit data with the primary user absent, or with the primary user present but miss detection happened. For the former case, the primary user may come back in the $S-R$ link, or come back in the $R-D$ link, or come back in the $S-R$ link but leave in the $R-D$ link. For the latter case, we have the similar three situations. In this section, we will discuss all the six situations in detail.

\section{A. Three Scenarios with Initially Absent Primary User}

1) Primary User Arrives in $S-R$ link: In this case, there is no primary user traffic at the beginning. The primary user comes at the $k_{1}^{\text {th }}$ symbol in the $S-R$ link, and keeps its presence in the whole $R-D$ link. The probability for this case is given by

$$
P_{f}\left\{k_{1}\right\}=p_{f} p_{00}^{k_{1}-1} p_{01} p_{11}^{M+N-k_{1}} .
$$

The whole $S-D$ link can be divided into three periods: (1) $0 \sim k_{1}^{\text {th }}$ symbols in the $S-R$ link without the primary user traffic; (2) $k_{1}^{\text {th }} \sim M$ symbols in the $S-R$ link with the primary user traffic; (3) the whole $R-D$ link with the primary user present. The $S-D$ link SNR, the instantaneous BER and average BER over these periods are given by

\begin{tabular}{|c|c|c|c|}
\hline Period & SNR & BER & $\overline{\text { BER }}$ \\
\hline$(1)$ & $\gamma$ & $\mathrm{BER}_{0}$ & $\mathrm{BER}_{0}$ \\
\hline$(2)$ & $\gamma_{A}^{\prime}$ & $\operatorname{BER}\left(\gamma_{A}^{\prime}, M\right)$ & $\overline{B E R}_{1}$ \\
\hline$(3)$ & $\gamma_{B}^{\prime}$ & $\operatorname{BER}\left(\gamma_{B}^{\prime}, M\right)$ & $\overline{\mathrm{BER}}_{2}$ \\
\hline
\end{tabular}

The average BER for the whole period is the average BER of each period weighted by the time that each period lasts

$\overline{\mathrm{BER}}_{f\left(k_{1}\right)}=\frac{\left(k_{1}-1\right) \mathrm{BER}_{0}+\left(M-k_{1}+1\right) \overline{\mathrm{BER}}_{1}+N \overline{\mathrm{BER}}_{2}}{M+N}$.

2) Primary User Arrives in $R-D$ link: Before the primary user comes at the $k_{2}^{\text {th }}$ symbol in the $R-D$ link, there is no primary user traffic in the $S-R$ link and the $1 \sim k_{2}^{\text {th }}$ symbols in the $R-D$ link. The probability this case happens is

$$
P_{f}\left\{k_{2}\right\}=p_{f} p_{00}^{M+k_{2}-1} p_{01} p_{11}^{N-k_{2}} .
$$

Similarly, the whole $S-D$ link can be divided into two periods: (1) the whole $S-R$ link and $0 \sim k_{2}^{\text {th }}$ symbols in the $R-D$ link before the primary user arrives; (2) $k_{2}^{\text {th }} \sim N$ symbols in the $R-D$ link after the primary user arrives, the statistics of which are given as 


$$
\begin{aligned}
\overline{\mathrm{BER}}=\sum_{k_{1}=1}^{M} \sum_{k_{2}=1}^{N} & \left(\left(1-P_{f a}\right)\left(\overline{\mathrm{BER}}_{f\left(k_{1}\right)} P_{f}\left\{k_{1}\right\}+\overline{\mathrm{BER}}_{f\left(k_{2}\right)} P_{f}\left\{k_{2}\right\}+\overline{\mathrm{BER}}_{f\left(k_{1}, k_{2}\right)} P_{f}\left\{k_{1}, k_{2}\right\}\right)\right. \\
& \left.+P_{m d}\left(\overline{\mathrm{BER}}_{b\left(k_{1}\right)} P_{b}\left\{k_{1}\right\}+\overline{\mathrm{BER}}_{b\left(k_{2}\right)} P_{b}\left\{k_{2}\right\}+\overline{\mathrm{BER}}_{b\left(k_{1}, k_{2}\right)} P_{b}\left\{k_{1}, k_{2}\right\}\right)\right)
\end{aligned}
$$

\begin{tabular}{|c|c|c|c|}
\hline Period & SNR & BER & $\overline{\text { BER }}$ \\
\hline$(1)$ & $\gamma$ & $\mathrm{BER}_{0}$ & $\mathrm{BER}_{0}$ \\
\hline$(2)$ & $\gamma_{B}^{\prime}$ & $\operatorname{BER}\left(\gamma_{B}^{\prime}, M\right)$ & $\overline{\mathrm{BER}}_{2}$ \\
\hline
\end{tabular}

In this case, the average BER for the whole period is thus given by

$$
\overline{\operatorname{BER}}_{f\left(k_{2}\right)}=\frac{\left(M+k_{2}-1\right) \mathrm{BER}_{0}+\left(N-k_{2}+1\right) \overline{\mathrm{BER}}_{2}}{M+N} .
$$

3) Primary User Arrives in $S-R$ link but Leaves in $R-D$ link: If the primary arrives at the $k_{1}^{\text {th }}$ symbol in the $S-R$ link but leaves at the $k_{2}^{\text {th }}$ symbol in the $R-D$ link, the probability of this case yields

$$
P_{f}\left\{k_{1}, k_{2}\right\}=p_{f} p_{00}^{k_{1}-1} p_{01} p_{11}^{M-k_{1}+k_{2}-1} p_{10} p_{00}^{N-k_{2}} .
$$

The whole $S-D$ link is divided into three periods: (1) $0 \sim k_{1}^{\text {th }}$ symbols in the $S-R$ link and $k_{2}^{\text {th }} \sim N$ symbols in the $R-D$ link without any primary user traffic; (2) $k_{1}^{\text {th }} \sim M$ symbols in the $S-R$ link with the primary user present; (3) $0 \sim k_{2}^{\text {th }}$ symbols in the $R-D$ link with the primary user present. The statistics for these periods are as follows:

\begin{tabular}{|c|c|c|c|}
\hline Period & SNR & BER & $\overline{\mathrm{BER}}$ \\
\hline$(1)$ & $\gamma$ & $\mathrm{BER}_{0}$ & $\mathrm{BER}_{0}$ \\
\hline$(2)$ & $\gamma_{A}^{\prime}$ & $\operatorname{BER}\left(\gamma_{A}^{\prime}, M\right)$ & $\overline{\mathrm{BER}}_{1}$ \\
\hline$(3)$ & $\gamma_{B}^{\prime}$ & $\operatorname{BER}\left(\gamma_{B}^{\prime}, M\right)$ & $\overline{\mathrm{BER}}_{2}$ \\
\hline
\end{tabular}

The average BER for the whole $S-D$ link period is

$$
\begin{aligned}
\overline{\mathrm{BER}}_{f\left(k_{1}, k_{2}\right)} & =\frac{\left(k_{1}+N-k_{2}\right) \mathrm{BER}_{0}}{M+N} \\
& \frac{\left(M-k_{1}+1\right) \overline{\mathrm{BER}}_{1}+\left(k_{2}-1\right) \overline{\mathrm{BER}}_{2}}{M+N} .
\end{aligned}
$$

\section{B. Three Scenarios with Initially Present Primary User}

1) Primary User Leaves in $S-R$ link: At the beginning, the primary user is present over the $S-R$ link, but it leaves at the $k_{1}^{\text {th }}$ symbol and keeps absent over the $R-D$ link. The probability of this case should be

$$
P_{b}\left\{k_{1}\right\}=p_{b} p_{11}^{k_{1}-1} p_{10} p_{00}^{N+M-k_{1}} .
$$

Similarly to the first case, the status of the $S-D$ period, which can also be divided into three periods, can be shown as

\begin{tabular}{|c|c|c|c|}
\hline Period & SNR & BER & $\overline{\text { BER }}$ \\
\hline$(1)$ & $\frac{\gamma}{1+\gamma_{p}}$ & $\mathrm{BER}_{0}$ & BER $_{0}$ \\
\hline$(2)$ & $\gamma_{B}^{\prime}$ & $\operatorname{BER}\left(\gamma_{B}^{\prime}, M^{\prime}\right)$ & $\overline{\mathrm{BER}}_{3}$ \\
\hline$(3)$ & $\gamma_{A}^{\prime}$ & $\operatorname{BER}\left(\gamma_{A}^{\prime}, M^{\prime}\right)$ & $\overline{\mathrm{BER}}_{4}$ \\
\hline
\end{tabular}

where the periods are (1) $0 \sim k_{1}^{\text {th }}$ symbols in the $S-R$ link with the primary user present; (2) $k_{1}^{\text {th }} \sim M$ symbols in the $S-R$ link with no primary user traffic; (3) the while $R-D$ link with no primary user traffic. Thus the average BER of the whole period is given by

$$
\overline{\mathrm{BER}}_{b\left(k_{1}\right)}=\frac{\left(k_{1}-1\right) \mathrm{BER}_{0}+\left(M-k_{1}+1\right) \overline{\mathrm{BER}}_{3}+N \overline{\mathrm{BER}}_{4}}{M+N} .
$$

2) Primary User Leaves in $R-D$ link: If the primary user is present in the whole $S-R$ link and leaves at the $k_{2}^{\text {th }}$ symbol in the $R-D$ link, the probability of its happening yields

$$
P_{b}\left\{k_{2}\right\}=p_{b} p_{11}^{M+k_{2}-1} p_{10} p_{00}^{N-k_{2}} .
$$

The status of the two divided periods, including (1) the whole $S-R$ link and $0 \sim k_{2}^{\text {th }}$ symbols in the $R-D$ link before the primary user leaves, and (2) $k_{2}^{\text {th }} \sim N$ symbols in the $R-D$ link after the primary user leaves, is given as

\begin{tabular}{|c|c|c|c|}
\hline Period & SNR & BER & $\overline{\text { BER }}$ \\
\hline$(1)$ & $\frac{\gamma}{1+\gamma_{p}}$ & BER $_{0}$ & BER $_{0}$ \\
\hline$(2)$ & $\gamma_{A}^{\prime}$ & $\operatorname{BER}\left(\gamma_{A}^{\prime}, M^{\prime}\right)$ & $\overline{\text { BER }}_{4}$ \\
\hline
\end{tabular}

And the the average BER of the whole period can be written as

$$
\overline{\mathrm{BER}}_{b\left(k_{2}\right)}=\frac{\left(M+k_{2}-1\right) \mathrm{BER}_{0}+\left(N-k_{2}+1\right) \overline{\mathrm{BER}}_{4}}{M+N} .
$$

3) Primary User Leaves in $S-R$ link and Arrives in $R-D$ link: In this case, the primary user leaves at the $k_{1}^{\text {th }}$ symbol in the $S-R$ link, after a short break, however, it returns at the $k_{2}^{\text {th }}$ symbol in the $R-D$ link. The probability this case happens is given as

$$
P_{b}\left\{k_{1}, k_{2}\right\}=p_{b} p_{11}^{k_{1}-1} p_{10} p_{00}^{M-k_{1}+k_{2}-1} p_{01} p_{11}^{N-k_{2}} .
$$

The status of the whole period can be divided into three periods as

\begin{tabular}{|c|c|c|c|}
\hline Period & $\mathrm{SNR}_{S-D}$ & BER & $\overline{\mathrm{BER}}$ \\
\hline$(1)$ & $\frac{\gamma}{1+\gamma_{p}}$ & $\mathrm{BER}_{0}$ & $\mathrm{BER}_{0}$ \\
\hline$(2)$ & $\gamma_{B}^{\prime}$ & $\operatorname{BER}\left(\gamma_{B}^{\prime}, M^{\prime}\right)$ & $\overline{\mathrm{BER}}_{3}$ \\
\hline$(3)$ & $\gamma_{A}^{\prime}$ & $\operatorname{BER}\left(\gamma_{A}^{\prime}, M^{\prime}\right)$ & $\overline{\mathrm{BER}}_{4}$ \\
\hline
\end{tabular}

where the periods are (1) $0 \sim k_{1}^{\text {th }}$ symbols in the $S-R$ link and $k_{2}^{\text {th }} \sim N$ symbols in the $R-D$ link under the presence of the primary user; (2) $k_{1}^{\text {th }} \sim M$ symbols in the $S-R$ link with the primary user absent; (3) $0 \sim k_{2}^{\text {th }}$ symbols in the $R-D$ link without the primary user. The average BER for the whole period can be written as

$$
\begin{aligned}
\overline{\mathrm{BER}}_{b\left(k_{1}, k_{2}\right)} & =\frac{\left(k_{1}+N-k_{2}\right) \mathrm{BER}_{0}}{M+N}+ \\
& \frac{\left(M-k_{1}+1\right) \overline{\mathrm{BER}}_{3}+\left(k_{2}-1\right) \overline{\mathrm{BER}}_{4}}{M+N} .
\end{aligned}
$$




\section{Average BER}

When there is no primary user at the beginning, the secondary user is allowed to transmit only if the free spectrum bands are detected, the probability of which is $1-P_{f a}$. On the other hand, the secondary user can transmit with primary user present only if the miss detection happens, the probability of which yields $P_{m d}$. The BER averaged over all possibilities of the six scenarios given above yields (39) shown on the top of this page.

\section{Numerical Results}

In this section, we present some numerical results to show the effect of the primary user traffic on the BER of the secondary user transmission. The false-alarm probability and miss-detection probability are fixed as $P_{f a}=0.1$ and $P_{m d}=$ 0.1 . The number of the data symbols for each link is chosen as $M=N=10$.

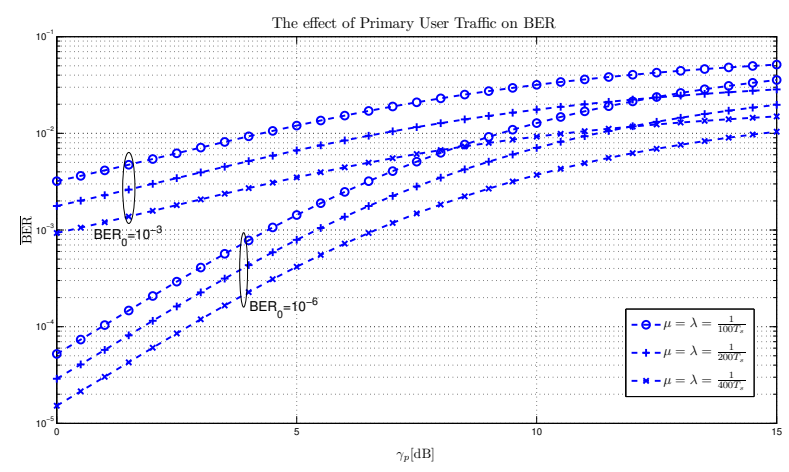

Figure 1: The average BER for different values of primary user traffic $\gamma_{p}\left(\bar{\gamma}_{1}=10 \mathrm{~dB}, \bar{\gamma}_{2}=5 \mathrm{~dB}\right.$, and $\left.m_{1}=3, m_{2}=5\right)$.

In Fig. 1, we examine the effect of the primary user traffic on the BER of the secondary user transmission. The channel parameters for $S-R$ link and $R-D$ link are chosen as $\bar{\gamma}_{1}=10 \mathrm{~dB}, \bar{\gamma}_{2}=5 \mathrm{~dB}$, and $m_{1}=3, m_{2}=5$. From the figure, we can see that the primary user traffic degrades the BER significantly, and the smaller target BER we want to maintain, the greater impact the primary user traffic will exert. Second, as the primary user traffic increases, the impact on the average SNR marginally decreases. In addition, the symbol time $T_{s}$ also affects the average BER. When $T_{s}$ is larger, the transmission time for each link lasts longer when the symbol number are fixed. In this way the sudden come or leave of the primary user is more likely to happen so that the impact of the primary user will be greater.

In Fig. 2, we show the average BER over different average SNRs of $S-R$ link and $R-D$ link. The Nakagami parameters are chosen as $m_{1}=3$ and $m_{2}=5$, and the primary user traffic is fixed as $\gamma_{p}=10 \mathrm{~dB}$. The parameter for the symbol time is $\lambda=\mu=\frac{1}{200 T_{s}}$. With the target BER as $\mathrm{BER}_{0}=10^{-3}$, we can see in this figure that $\bar{\gamma}_{1}$ has a greater influence on the BER than $\bar{\gamma}_{2}$. Generally, the primary user is more likely to be present over the $R-D$ link, whose average BER is given

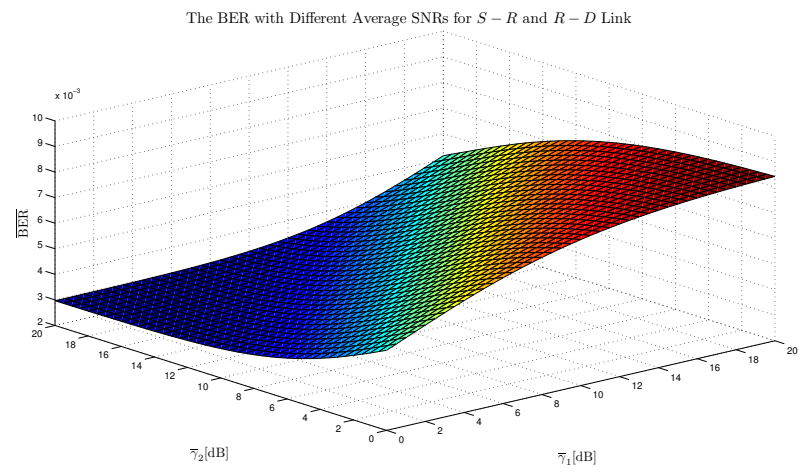

Figure 2: The average BER for different values of the average SNRs of $S-R$ link and $R-D$ link $\left(m_{1}=3, m_{2}=5\right.$, and $\left.\gamma_{p}=10 \mathrm{~dB}\right)$.

by (19) and (26). By examining (19) and (26), we can find that the random variable $x_{B}$ in (18) plays an important role, in which the $S-R$ link SNR $\gamma_{1}$ have a greater weight over the $R-D$ link SNR $\gamma_{2}$.

\section{CONCLUSION}

In this paper, we examined the impact of the presence of the primary user on the performance of data transmission of the secondary user. With a relay, six scenarios of the primaryuser change have been analyzed, and we give the average BER for each situation, as well as the average BER over all possible situations. Not affecting the link spectral efficiency, the primary user traffic can degrade the BER considerably, and the smaller the target BER we want to maintain, the more sensitive the average BER will be.

\section{REFERENCES}

[1] I. Akyildiz, W. Lee, M. Vuran, and S. Mohanty, "NeXt generation/dynamic spectrum access/cognitive radio wireless networks: A survey," Computer Networks, vol. 50, no. 13, pp. 2127-2159, September, 2006.

[2] J. Laneman, D. Tse, and G. Wornell, "Cooperative diversity in wireless networks: Efficient protocols and outage behavior," IEEE Transactions on Information Theory, vol. 50, no. 12, pp. 3062-3080, December, 2004.

[3] G. Ganesan and Y. Li, "Cooperative spectrum sensing in cognitive radio, part i: Two user networks," IEEE Transactions on Wireless Communications, vol. 6, no. 6, pp. 2204-2213, June, 2007.

[4] - "Cooperative spectrum sensing in cognitive radio, part ii: Multiuser networks," IEEE Transactions on Wireless Communications, vol. 6, no. 6, pp. 2214-2222, 2007.

[5] A. J. Goldsmith and S. G. Chua, "Variable-rate variable-power MQAM for fading channels," IEEE Transactions on Communications, vol. 45, no. 10, pp. 1218-1230, October, 1997.

[6] M. Nakagami, "The m-distribution- A general formula of intensity distribution of rapid fading," in Statistical methods in radio wave propagation (W. G. Hoffman, ed.), pp. 3-36, Pergamon Press, Oxford, England, 1960.

[7] M. Torabi, W. Ajib, and D. Haccoun, "Performance analysis of amplifyand-forward cooperative networks with relay selection over rayleigh fading channels," in Proc.Vehicular Technology Conference (VTC'09, Spring), pp.1-5, Barcelona, Spain, April, 2009.

[8] A. Papoulis, S. Pillai, and S. Unnikrishna, Probability, random variables, and stochastic processes, McGraw-Hill, New York, 1965.

[9] I. S. Gradshteyn and I. M. Ryzhik, Table of Integrals, Series, and Products, San Diego, CA: Academic Press, fifth ed., 1994. 